INPLASY

PROTOCOL

To cite: Leonardo et al. Biofeedback Pelvic Floor Muscle Training and Pelvic Electrical Stimulation in Women with Overactive Bladder: A Systematic Review and Meta-analysis of

Randomized Controlled Trials. Inplasy protocol 202220024. doi:

10.37766/inplasy2022.2.0024

Received: 09 February 2022

Published: 09 February 2022

Corresponding author:

Kevin Leonardo

kevinleonardosoputro@gmail.com

Author Affiliation:

Division of Urology,

Department of Surgery,

Persahabatan Hospital, DKI

Jakarta, Indonesia.

Support: This study received no funding.

Review Stage at time of this submission: Data analysis -

Completed but not published.

Conflicts of interest:

None declared.

\section{Biofeedback Pelvic Floor Muscle Training and Pelvic Electrical Stimulation in Women with Overactive Bladder: A Systematic Review and Meta-analysis of Randomized Controlled Trials}

Leonardo, $\mathrm{K}^{1}$; Seno, $\mathrm{DH}^{2}$; Mirza, $\mathrm{H}^{3}$; Afriansyah, $\mathrm{A}^{4}$.

Review question / Objective: Population : Overactive Bladder, Women; Intervention : Biofeedback assisted PFMT and/or pelvic Electrical Stimulation with non-implanted electrodes (on the skin surface around perianal, intra vaginal or rectal); Comparison : PFMT only / Bladder Training / Life style modification-recommendation; Outcome : Changes in Quality of life, Incontinence Episodes, Number of participant cured/ improved.

Condition being studied: Overactive Bladder syndrome which has been defined as urinary urgency. It is not life threatening disease, therefore, often ignored by patients, but the effect in daily life can be very bothersome.

INPLASY registration number: This protocol was registered with the International Platform of Registered Systematic Review and Meta-Analysis Protocols (INPLASY) on 09 February 2022 and was last updated on 09 February 2022 (registration number INPLASY202220024).

\section{INTRODUCTION}

Review question / Objective: Population : Overactive Bladder, Women; Intervention : Biofeedback assisted PFMT and/or pelvic
Electrical Stimulation with non-implanted electrodes (on the skin surface around perianal, intra vaginal or rectal); Comparison : PFMT only / Bladder Training / Life style modification- 
recommendation; Outcome : Changes in Quality of life, Incontinence Episodes, Number of participant cured/improved.

Rationale: There was no clear description of the effects of Biofeedback assisted PFMT and pelvic ES as the treatment of OAB. The major goal of this study was to look at the role and efficacy of interventions in terms of improving QoL and reducing symptoms following therapy.

Condition being studied: Overactive Bladder syndrome which has been defined as urinary urgency. It is not life threatening disease, therefore, often ignored by patients, but the effect in daily life can be very bothersome.

\section{METHODS}

Search strategy: Literature searches were undertaken in five databases (PubMed, Cochrane, CINAHL, EMBASE, Scopus) up to November 2021 using the search queries of ("Overactive bladder" OR "Incontinence") AND ("Biofeedback" OR "electrical stimulation") AND ("pelvic floor muscle training" OR "bladder training").

Participant or population: Women with Overactive Bladder.

Intervention: Biofeedback assisted pelvic floor muscle training (PFMT) and/or pelvic electrical stimulation (non-implanted electrode around pelvic area - on the skin surface/transcutaneousaround perianal, intra vaginal or rectal).

Comparator: Conventional conservative therapy such as pelvic floor muscle training (PFMT) only / Bladder Training / Lifestyle modification-recommendation.

\section{Study designs to be included: RCTs.}

Eligibility criteria: [1] women with diagnosis of OAB, [2] intervention group either biofeedback or pelvic electrical stimulation (non-implanted electrode around pelvic area (on the skin surface/transcutaneous around perianal, intra vaginal or rectal) , [3] the evaluated outcomes should in terms include at least one of these parameters : QoL changes, incontinence episodes and symptom reduction/improvement after the intervention was given as the conservative treatment.

Information sources: Mostly from electronic database such as PubMed, Cochrane, CINAHL, EMBASE, Scopus.

Main outcome(s): QoL changes -measures of effect : Standardized mean difference (SMD).

Additional outcome(s): Incontinence episodes and symptoms reduction/ improvement (number of participant) -measures of effect : Standardized mean difference (SMD) and Relative Risks.

Data management: The literature searches and selection were performed independently by all of the authors. If there are differences from the screening, the reviewers conducted the discussion. Mendeley Reference Manager was used to identify and eliminate duplicate records from different databases.

Quality assessment / Risk of bias analysis: Quality Assessment tools that will be used is the Cochrane Risk of Bias Tool assessing selection bias, detection bias, performance bias, reporting bias, and attrition bias.

Strategy of data synthesis: The selection and inclusion of studies will be performed in two stages by two independent reviewers. The retrieved records are first screened on the basis of the titles/ abstracts, and then the full-text papers are examined for eligibility. Information needed from the articles was retrieved and collected into a table. For the studies that provide relevant outcome data, the metaanalysis was conducted using the Cochrane Collaboration Review Manager (Rev-Man version 5.4). To identify the heterogeneity between studies $I^{2}$ were used. The study considers being heterogenous if the $I^{2} 50 \%$. When significant heterogeneity was observed, the random-effects model was used to combine the effect sizes of the included 
studies; otherwise, the fixed effects model was adopted.

Subgroup analysis: There is no subgroups analysis planned so far.

Sensitivity analysis: By far, we conduct sensitivity analysis in Rev-Man by repeating the meta-analysis multiple times, each time leaving out one sample.

Language: English.

Country(ies) involved: Indonesia.

Other relevant information: This is a review that involves several recent studies compared to the previous one. The intervention group that compared to the control group include a quite specific treatment which is pelvic electrical stimulation, that makes a difference compared to the other reviews.

Keywords: overactive bladder; biofeedback; electrical stimulation; pelvic floor muscle training.

Contributions of each author:

Author 1 - Kevin Leonardo - data acquisition and analysis, drafted the manuscript.

Email: kevinleonardosoputro@gmail.com

Author 2 - Doddy Hami Seno - Study concept and design, Critical revision of the manuscript.

Email: doddy.uro@gmail.com

Author 3 - Hendy Mirza - Study concept and design, Critical revision of the manuscript.

Email: hendyuro@gmail.com

Author 4 - Andika Afriansyah - data acquisition and analysis, drafted the manuscript.

Email: andikaafriansyah@gmail.com 